\title{
Autoeficacia General Percibida en Universitarios Mexicanos, Diferencias entre Hombres y Mujeres
}

\author{
Juan F. Aguirre, José R. Blanco, Judith M. Rodríguez-Villalobos, Martha Ornelas* \\ Universidad Autónoma de Chihuahua. Facultad de Ciencias de la Cultura Física. DES Salud CAs 101 y \\ 121. Calle Escorza 900, CP 31000 Chihuahua, Chih.- México. (e-mail: jaguirre@uach.mx, \\ jblanco@uach.mx, jurodrig@uach.mx y mornelas@uach.mx) \\ * Autor a quien debe ser dirigida la correspondencia
}

Recibido Mar. 4, 2015; Aceptado Abr. 10, 2015; Versión final May. 7, 2015, Publicado Oct. 2015

\begin{abstract}
Resumen
El objetivo de esta investigación consistió comparar los perfiles de autoeficacia general percibida de mujeres y hombres universitarios del noroeste de México. La muestra total fue de 282 sujetos; 142 mujeres y 140 hombres, con una edad media de 20 y 21 años, respectivamente. El abordaje adoptado en la investigación se enmarcó dentro de un enfoque cuantitativo con un diseño descriptivo tipo encuesta. Los resultados del análisis multivariante de varianza, seguido por los análisis de varianza univariados, muestran que las mujeres en comparación con los hombres reportan puntuaciones más altas en autoeficacia percibida y con mayor necesidad y posibilidad de ser más autoeficaces. Las diferencias encontradas entre hombres y mujeres con respecto a su percepción de autoeficacia, sugieren que al diseñar cualquier tipo de intervención que tenga como objetivo la mejora de la autoeficacia percibida habrá que tomar en cuenta a la variable género.
\end{abstract}

\section{General Perceived Self-Efficacy in Mexican University Students, Differences between Men and Women}

\begin{abstract}
The aim of the present study was to compare the profiles of general perceived self-efficacy between men and women mexican university students. A total sample of 282 participants, 142 women and 140 men with a mean age of 20 and 21 years, respectively, participated in this study. A quantitative approach with a descriptive and transversal survey design was applied. The results of the one-way multivariate analysis of variance, followed by the one-way univariate analyses of variance, showed that women reported statistically significant higher punctuations than men regarding the perceived self-efficacy and greatest need and ability to be more self-efficacious. Differences found between men and women regarding their perceived selfefficacy, suggest that any effort to improve perceived self-efficacy must take gender into consideration.
\end{abstract}

Keywords: student's beliefs, higher education, self-efficacy, student characteristics 


\section{INTRODUCCIÓN}

La autoeficacia se define como "los juicios de las personas acerca de sus capacidades para alcanzar niveles determinados de rendimiento" (Bandura, 1997). Por tanto, no basta con ser capaz de, es preciso juzgarse capaz. Capaz de utilizar las habilidades y destrezas personales ante circunstancias muy diversas, incluso en las reacciones emocionales que se experimentan ante situaciones difíciles (Ornelas et al., 2012; Schmidt et al., 2008). La percepción de las personas acerca de su propia eficacia se alza como un requisito fundamental para desarrollar con éxito las acciones conducentes al logro de los objetivos personales (Salanova et al., 2011). Dicha autopercepción, ejerce una profunda influencia en la elección de tareas y actividades, en el esfuerzo y perseverancia de las personas cuando se enfrentan a determinados retos e incluso en las reacciones emocionales que experimentan ante situaciones difíciles. En definitiva, las creencias de autoeficacia representan un mecanismo cognitivo que media entre el conocimiento y la acción y que determina, junto con otras variables, el éxito de las propias acciones (Gutiérrez et al., 2011).

Al hablar de autoeficacia, en general, se hace referencia a la eficacia percibida en una situación concreta (Bandura, 1986). Sin embargo, la autoeficacia también puede entenderse en sentido amplio, como un constructo global referido a la creencia estable que tiene un individuo sobre su capacidad para manejar de manera adecuada una amplia variedad de estresores de la vida cotidiana y la Escala de Autoeficacia General (EAG) se encuentra enmarcada dentro de este sentido amplio del constructo de autoeficacia y evalúa la percepción de competencia personal para manejar de forma eficaz una gran variedad de situaciones estresantes. Como una muestra de la importancia de la autoeficacia podemos decir que esta revela por qué las personas con el mismo nivel de habilidad y conocimiento presentan conductas y/o resultados diferentes, o por qué las personas actúan en disonancia con sus habilidades (Bandura, 1982; Pérez et al., 2011). Lo anterior se explica porque el rendimiento académico adecuado también depende de la eficacia percibida para manejar exitosamente demandas académicas. Por ello, la percepción de autoeficacia es imprescindible para dominar las actividades académicas; ya que los estudiantes que confían en sus capacidades se sienten más motivados para alcanzar sus metas (Á. Blanco, 2010; Rodríguez, 2009).

Desde la Teoría del Aprendizaje Social de Bandura se asume entonces que las expectativas de autoeficacia son un importante predictor de las intenciones y acciones de los individuos frente a diversas situaciones; ya que un elevado nivel de autoeficacia percibida se ha mostrado como un elemento protector que hace aumentar la motivación, disminuir las alteraciones emocionales, al tiempo que mejora las conductas saludables en el cuidado físico. De hecho, frente a lo difícil que puede ser motivar a la adopción de conductas que promuevan la salud o detener conductas nocivas para ésta, la autoeficacia ha mostrado consistentemente ser un factor de gran importancia (Olivari y Urra, 2007). La autoeficacia percibida pues, desempeña un papel fundamental en el funcionamiento humano puesto que afecta el comportamiento no sólo de manera directa, sino también por su impacto en otros determinantes claves tales como metas y aspiraciones, expectativas de resultados, tendencias afectivas y percepción de los impedimentos y oportunidades que se presentan en el medio social (Bandura, 1997).

Por todo lo antes expuesto, las creencias de autoeficacia influyen en los pensamientos de las personas, en los cursos de acción que ellas eligen para perseguir, los desafíos y metas que ellas se plantean para sí mismas y su compromiso con los mismos, la cantidad de esfuerzo que invierten en determinadas tareas; los resultados que esperan alcanzar por sus esfuerzos, la magnitud de su perseverancia frente a los obstáculos, su resistencia a la adversidad, el nivel de estrés y depresión que experimentan cuando se enfrentan con demandas exigentes del ambiente y los logros que alcanzan (Sansinenea et al., 2008).

Este trabajo es fundamentalmente un estudio de tipo descriptivo que intenta comparar los perfiles de autoeficacia general percibida de hombres y mujeres universitarios mexicanos y pretende, como investigación aplicada, aportar información para el logro de una práctica educativa de mayor calidad en el contexto de atención a la diversidad; contribuyendo al saber pedagógico en el esclarecimiento de los factores que conforman un modelo rendimiento escolar y desarrollo humano integral; bajo la premisa de que, darse a la tarea de mejorar la percepción de ser capaz en quien aprende es un objetivo educacional valioso, bajo el supuesto implícito de que su potenciación servirá como vehículo para la mejora de otros resultados tales como el logro académico y la autoestima.

\section{METODOLOGÍA}

\section{Participantes}

La muestra de 282 participantes, $142(50.4 \%)$ mujeres y $140(49.6 \%)$ hombres, se obtuvo mediante un muestreo por conveniencia. Las edades de las mujeres fluctúan entre los 18 y 24 años, con una media de 
20.35 y una desviación estándar de 1.57 años; y las de los hombres fluctúan entre los 18 y 24 años, con una media de 20.92 y una desviación estándar de 1.77 años.

\section{Instrumento}

Escala de Autoeficacia General de Baessler y Schwarzer (EAG) adaptada por Sanjuán et al. (2000), evalúa la percepción de competencia personal para manejar de forma eficaz una gran variedad de situaciones estresantes (Tabla 1). La versión es español del cuestionario aplicado a universitarios españoles obtuvo una consistencia interna aceptable; $\alpha=.87$ (Sanjuán et al., 2000). Para nuestro estudio se hicieron dos nuevas adaptaciones:

Tabla 1: Reactivos de Escala de Autoeficacia General.

\begin{tabular}{|ll|}
\hline Reactivo \\
\hline 1 & Encontrar la manera de obtener lo que quiero aunque alguien se me oponga \\
\hline 2 & Resolver problemas difíciles si me esfuerzo lo suficiente \\
\hline 3 & Persistir en lo que me he propuesto hasta llegar a alcanzar mis metas \\
\hline 4 & Manejar eficazmente acontecimientos inesperados \\
\hline 5 & Superar situaciones imprevistas \\
\hline 6 & Permanecer tranquilo cuando me encuentro en dificultades, porque cuento con las habilidades \\
\hline 7 & Salir triunfador venga lo que venga \\
\hline 8 & Resolver la mayoría de los problemas a los que me enfrento \\
\hline 9 & Planear qué debo hacer si me encuentro en una situación difícil \\
\hline 10 & Generar varias alternativas de solución al tener que hacer frente a un problema \\
\hline
\end{tabular}

Primera adaptación, en la escala original se puntúa con cinco opciones de respuesta, en la versión utilizada en la presente investigación, el sujeto elige entre once opciones (desde 0 nada capaz hasta 10 absolutamente capaz), esta primer adaptación se justifica en relación a que los sujetos están acostumbrados a la escala de 0 a 10, ya que así han sido evaluados por el sistema educativo de nuestro país (México). Viciana et al. (2007) reportan un cambio similar en la validación de una escala con población española.

Segunda adaptación, de acuerdo a H. Blanco, Ornelas, Rueda, et al. (2013) en nuestra versión utilizamos tres escenarios de respuesta para cada uno de los ítems del cuestionario (Tabla 1): (a) Escenario de capacidad percibida, respondiendo en el contexto: que tan capaz me siento para...; (b) Escenario de interés en ser capaz, respondiendo en el contexto: que tanto interés tengo en ser capaz de...; y (c) Escenario de cambio en ser capaz de, respondiendo en el contexto: si me esfuerzo en cambiar, que tan capaz sería para... (Figura 1).

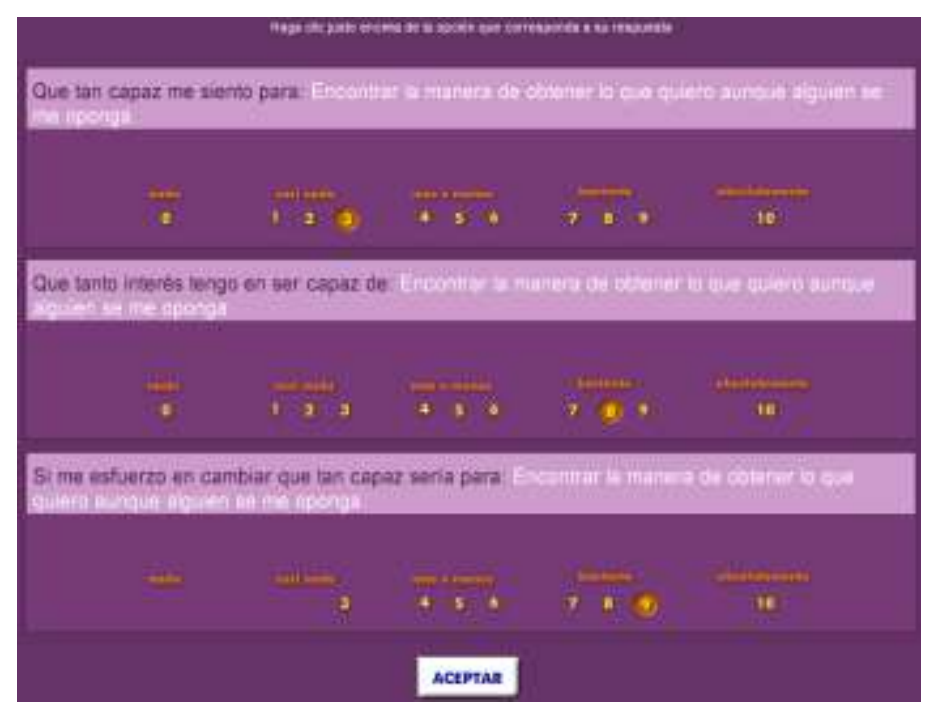

Fig. 1 Escenarios de respuesta para cada uno de los ítems de la escala. 
Para luego, a partir de sus respuestas obtener 4 índices: (1) Autoeficacia percibida.- obtenida a partir de las respuestas al escenario de capacidad percibida; (2) Autoeficacia deseada.- obtenida a partir de las respuestas al escenario interés en ser capaz; (3) Autoeficacia alcanzable.- obtenida a partir de las respuestas al escenario de cambio en ser capaz de; y (4) Posibilidad de mejoría en la autoeficacia percibida.- obtenida a través de la diferencia entre el índice 3 y 1 (Autoeficacia alcanzable menos Autoeficacia percibida).

\section{Diseño}

En cuanto al diseño del estudio, se utilizó un enfoque cuantitativo con un diseño descriptivo y transversal tipo encuesta (Hernández et al., 2010). La variable independiente fue el Género y las variables dependientes el promedio de las puntuaciones obtenidas en los cuatro índices de autoeficacia general.

\section{Procedimiento}

Una vez conseguido el permiso de las autoridades educativas correspondientes, se invitó a participar en el estudio a hombres y mujeres estudiantes universitarios de la ciudad de La Paz Baja California Sur México; los que aceptaron participar firmaron la carta de aceptación correspondiente. Luego se aplicó el instrumento antes descrito por medio de una computadora personal utilizando el módulo administrador del instrumento del editor de escalas versión 2.0 (H. Blanco, Ornelas, Tristán, et al., 2013), en una sesión de aproximadamente 30 minutos. Al inicio de cada sesión se hizo una pequeña introducción sobre la importancia de la investigación y de cómo acceder al instrumento; las instrucciones de como responder se encontraban en las primeras pantallas; antes del primer reactivo del instrumento. Al término de la sesión se les agradeció su participación. Una vez aplicado el instrumento se procedió a recopilar los resultados por medio del módulo generador de resultados del editor de escalas versión $2.0(\mathrm{H}$. Blanco, Ornelas, Tristán, et al., 2013).

\section{Análisis de datos}

Se realizaron análisis estadísticos descriptivos (medias y desviaciones estándar) para todas las variables. Posteriormente, después de verificar que los datos cumplían con los supuestos de los análisis estadísticos paramétricos, se llevó a cabo un análisis multivariante de varianza (MANOVA), seguido por los análisis de varianza univariados (ANOVAs), para examinar las diferencias entre mujeres y hombres en cuanto a su autoeficacia percibida. Por otra parte, el tamaño del efecto se estimó mediante el eta cuadrado $\left(\eta^{2}\right)$. La fiabilidad o consistencia interna de la cada variable se estimó utilizando el coeficiente alfa de Cronbach con un intervalo de confianza del 95\%. Todos los análisis estadísticos se realizaron con el programa SPSS versión 20.0 para Windows. El nivel de significación estadística se estableció en $p=0.05$.

\section{RESULTADOS Y DISCUSIÓN}

La Tabla 2 muestra los valores de las medias y las desviaciones estándar de la autoeficacia en autoeficacia general percibida, así como los resultados del MANOVA y los subsiguientes ANOVAs. En la Tabla Valores descriptivos se presentan como media (desviación estándar). Los resultados del MANOVA indicaron diferencias significativas globales de acuerdo a la variable género en las puntuaciones de autoeficacia general (Wilks' $\lambda=.946 ; p<.001 ; \eta^{2}=.054$ ). Posteriormente, los resultados de los ANOVAs mostraron que las mujeres reportaron mayor autoeficacia percibida $(F=9.836, p<.01)$, deseada $(F=8.830, p<.01)$ y alcanzable $(F=15.425, p<.001)$ que los hombres y sin diferencias significativas en la posibilidad de mejoría en su autoeficacia percibida. Finalmente, la consistencia interna (confiabilidad) de variables dependientes (índices) en el presente estudio fue muy alta: autoeficacia percibida .928 (.915-.940), autoeficacia deseada $.916(.901-.930)$ y autoeficacia alcanzable $.933(.921-.944)$.

Tabla 2: Resultados MANOVA y ANOVAs para las diferencias entre mujeres y hombres universitarios en los cuatro índices de autoeficacia general percibida.

\begin{tabular}{|l|c|c|c|c|c|}
\hline & $\begin{array}{c}\text { Mujeres } \\
(\mathrm{n}=142)\end{array}$ & $\begin{array}{c}\text { Hombres } \\
(\mathrm{n}=140)\end{array}$ & $F$ & $p$ & $\eta^{2}$ \\
\hline & & & 5.323 & $<.001$ & .054 \\
\hline Autoeficacia percibida & $7.699(1.345)$ & $7.135(1.660)$ & 9.839 & $<.01$ & .034 \\
\hline Autoeficacia deseada & $8.736(1.071)$ & $8.328(1.112)$ & 8.830 & $<.01$ & .034 \\
\hline Autoeficacia alcanzable & $9.282(0.903)$ & $8.760(1.296)$ & 15.425 & $<.001$ & .052 \\
\hline Posibilidad de mejoría & $1.583(1.089)$ & $1.625(1.120)$ & 0.102 & $>.05$ & .000 \\
\hline
\end{tabular}


Con respecto a las conductas estudiadas, se destaca que, en cuanto a la percepción de competencia personal para manejar de forma eficaz una gran variedad de situaciones estresantes, las mujeres en comparación con los hombres se perciben como más autoeficaces, con mayor necesidad y posibilidad de ser más autoeficaces; resultados que en general concuerda con los obtenidos por Flores et al. (2014) y Peinado et al. (2014) en estudios similares sobre diferencias de género en la percepción de autoeficacia.

Por otro lado las diferencias encontradas entre hombres y mujeres pueden ser explicadas de acuerdo a la teoría social cognitiva (Bandura, 1999), según la cual las expectativas de autoeficacia son uno de los principales condicionantes de las diferencias de género en la toma de decisiones, diferencias que son resultado del proceso de socialización que da lugar a que hombres y mujeres tengan una percepción diferente acerca de las tareas, actividades y ocupaciones que son más apropiadas para cada género.

Sin embargo, consideramos que más estudios son necesarios con el fin de corroborar o refutar los datos obtenidos en la presente investigación. Dos limitaciones están presentes en este trabajo. La primera es que los participantes son solo estudiantes universitarios mexicanos, lo que supone una amenaza para la posibilidad de generalizar estos resultados. Ampliar la muestra (agregando por estudiantes de los otros niveles educativos) es un área de trabajo de cara al futuro. La segunda limitación proviene del propio instrumento de evaluación, que se basa en el autoinforme y que puede contener los sesgos que se derivan de la deseabilidad social.

\section{CONCLUSIONES}

De los resultados mostrados, de su análisis y de su discusión, se pueden obtener las siguientes conclusiones:

1) Las diferencias encontradas entre hombres y mujeres con respecto a su percepción de autoeficacia sugieren que al diseñar cualquier tipo de intervención que tenga como objetivo la mejora de la autoeficacia percibida habrá que tomar en cuenta a la variable género.

2) Tomando en cuenta que la investigación empírica ha demostrado, de manera amplia, que la autoeficacia resulta ser más predictiva del rendimiento académico que otras variables cognitivas (Bandura, 1982), que logra predecir el éxito posterior (Bandura, 1997) y que es un importante mediador cognitivo de competencia y rendimiento (Vera et al., 2011) en cuanto favorece los procesos cognoscitivos (Carbonero y Merino, 2008; Ornelas et al., 2011) se concluye que darse a la tarea de mejorar la percepción de ser capaz en quien aprende es un objetivo educacional valioso, bajo el supuesto implícito de que su potenciación servirá como vehículo para la mejora de otros resultados tales como el logro académico y la autoestima. Tomando en cuenta que la continua conciencia de fracaso reduce las expectativas de éxito y no favorece en ningún modo ni el aprendizaje ni el desarrollo personal.

Se subraya además la importancia de realizar un mayor número de investigaciones sobre el tema en nuestro país, ya que casi todos los estudios sobre el mismo han sido realizados en otros países.

\section{REFERENCIAS}

Bandura A., Self-efficacy mechanism inhuman agency, American Psychologist, 37(2), 122-147, (1982)

Bandura A., Pensamiento y acción. Fundamentos sociales, Alianza, Madrid, (1986)

Bandura A., Self-efficacy: The exercise of Control, Freeman, New York, (1997)

Bandura A., Autoeficacia: como afrontamos los cambios de la sociedad actual, Desclée de Brouwer, S. A., Madrid, (1999)

Blanco Á., Creencias de autoeficacia de estudiantes universitarios: un estudio empírico sobre la especificidad del constructo, RELIEVE, 16(1), 1-28, (2010)

Blanco H., Ornelas M., Rueda M. B., Martínez M., Composición factorial de la escala de autoeficacia en conductas académicas en universitarios de ciencias sociales, Revista Mexicana de Psicología, 30(1), 79-88, (2013)

Blanco H., Ornelas M., Tristán J. L., Cocca A., Mayorga-Vega D., López-Walle J., et al., Editor for creating and applying computerise surveys, doi: http://dx.doi.org/10.1016/j.sbspro.2013.12.105, Procedia Social and Behavioral Sciences, 106, 935-940, (2013) 
Carbonero M. Á., Merino E., Autoeficacia y madurez vocacional, Psicothema, 16(2), 229-234, (2008)

Flores F. J., Mayorga-Vega D., Blanco J. R., Blanco H., Perceived Self-Efficacy in Problem Solving and Scientific Communication in University Students. A Gender Study, doi: http://dx.doi.org/10.4236/psych.2014.55046, Psychology, 5, 358-364, (2014)

Gutiérrez M., Ampara E., Carminal P., Relaciones entre empatía, conducta prosocial, agresividad, autoeficacia y responsabilidad personal y social de los escolares, Psicothema, 23(1), 13-19, (2011)

Hernández R., Fernández C., Baptista P., Metodología de la investigación, McGraw- Hill, México, (2010)

Olivari C., Urra E., Autoeficacia y conductas de salud, Ciencia y Enfermería, 13(1), 9-15, (2007)

Ornelas M., Blanco H., Gastélum G., Chávez A., Autoeficacia Percibida en la conducta Académica de Estudiantes Universitarias, doi: 10.4067/S0718-50062012000200003, Formación Universitaria, 5(2), 17-26, (2012)

Ornelas M., Blanco H., Rodríguez J. M., Flores F. J., Análisis psicométrico de la escala autoeficacia en conductas de cuidado de la salud física en universitarios de primer ingreso, doi: 10.4067/S071850062011000600004, Formación Universitaria, 4(6), 21-34, (2011)

Peinado J. E., Viciana J., Blanco J. R., Blanco H., Perceived self-efficacy in the sociocultural sphere with mexican university students. Differences between men and women, European Scientific Journal, 10(8), 5968, (2014)

Pérez E., Lescano C., Heredia D., Zalazar P., Furlám L., Martínez M., Desarrollo y análisis psicométricos de un inventario de autoeficacia para inteligencias múltiples en niños argentinos Psicoperspectivas, 10(1), 169189, (2011)

Rodríguez M. N., Análisis factorial confirmatorio de la versión uruguaya de la escala Smart de Trapnell para medir capacidad intelectual percibida, Revista Iberoamericana de Diagnóstico y Evaluación Psicológica, 1(27), 85-105, (2009)

Salanova M., Llorens S., Shaufeli W. B., "Yes, I can, I feel good, and I just do it!" On gain cycles and spirals of efficacy beliefs, affect, and engagement, Applied Psychology: An International Review, 60(2), 255-285, (2011)

Sanjuán P., Pérez A. M., Bermúdez J., Escala de autoeficacia general: datos psicométricos de la adaptación para población española, Psicothema, 12(2), 509-513, (2000)

Sansinenea E., Gil L., Agirrezabal A., Larrañaga M., Ortiz G., Valencia J. F., et al., Autoconcordancia y autoeficacia en los objetivos personales: ¿Cuál es su aportación al bienestar?, Anales de Psicología, 24(1), 121-128, (2008)

Schmidt V., Messoulam N., Molina F., Autoconcepto académico en adolescentes de escuelas medias: presentación de un instrumento para su evaluación, Revista lberoamericana de Diagnóstico y Evaluación Psicológica, 1(25), 81-106, (2008)

Vera M., Salanova M., Martín-del-Río B., Self-efficacy among university faculty: how to develop an adjusted scale, Anales de Psicología, 27(3), 800-807, (2011)

Viciana J., Cervelló E. M., Ramírez J., Effects of manipulating positive and negative feedback on goal orientation, perceived motivational climate, satisfaction, task choice, perception of ability, and attitude to physical education lessons, Perceptual and motor skills, 105(1), 67-82, (2007) 